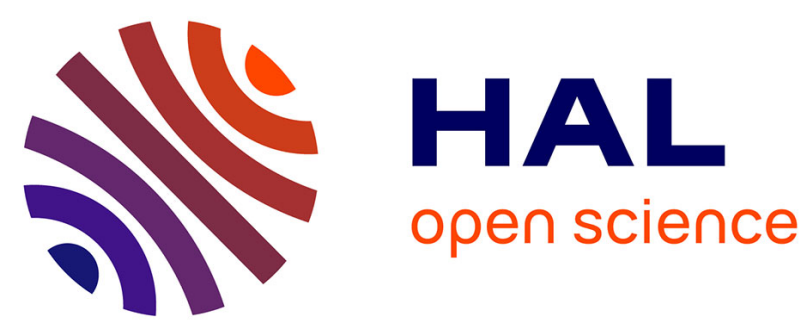

\title{
Safran-Isba-Modcou (SIM) : Un outil pour le suivi hydrométéorologique opérationnel et les études
}

Jean-Michel Soubeyroux, Eric Martin, Laurent Franchisteguy, Florence Habets, Joël Noilhan, Martine Baillon, Fabienne Regimbeau, Jean-Philippe Vidal, Patrick Le Moigne, Sophie Morel

\section{To cite this version:}

Jean-Michel Soubeyroux, Eric Martin, Laurent Franchisteguy, Florence Habets, Joël Noilhan, et al.. Safran-Isba-Modcou (SIM) : Un outil pour le suivi hydrométéorologique opérationnel et les études. La Météorologie, 2008, 63, pp.PP. 40-45. meteo-00350048

\section{HAL Id: meteo-00350048}

\section{https://hal-meteofrance.archives-ouvertes.fr/meteo-00350048}

Submitted on 4 Jun 2021

HAL is a multi-disciplinary open access archive for the deposit and dissemination of scientific research documents, whether they are published or not. The documents may come from teaching and research institutions in France or abroad, or from public or private research centers.
L'archive ouverte pluridisciplinaire HAL, est destinée au dépôt et à la diffusion de documents scientifiques de niveau recherche, publiés ou non, émanant des établissements d'enseignement et de recherche français ou étrangers, des laboratoires publics ou privés. 


\title{
Safran-Isba-Modcou (SIM)
}

\section{Un outil pour le suivi hydrométéorologique opérationnel et les études}

\author{
Jean-Michel Soubeyroux ${ }^{(1)}$, Éric Martin ${ }^{(2)}$, Laurent Franchisteguy(1), \\ Florence Habets ${ }^{(3)}$, Joël Noilhan ${ }^{(2)}$, Martine Baillon ${ }^{(1)}$, \\ Fabienne Regimbeau ${ }^{(1)}$, Jean-Philippe Vidal ${ }^{(1,2)}$, Patrick Le Moigne ${ }^{(2)}$ \\ et Sophie Morel ${ }^{(1,2,4)}$ \\ (1) Météo-France - Direction de la Climatologie \\ 42, avenue Gaspard-Coriolis - 31057 Toulouse Cedex 1 \\ (2) CNRM-Game (Météo-France, CNRS) \\ (3) UMR Sisyphe (Université Paris 6, CNRS) \\ (4) Metnext
}

\section{Résumé}

L'application Safran-Isba-Modcou, qui combine un système d'analyse de forçage atmosphérique, un schéma de surface et un module hydrogéologique, a fait l'objet de nombreux travaux de recherche au cours des quinze dernières années. Depuis 2003, ce modèle a été porté dans l'environnement opérationnel de Météo-France et s'est enrichi progressivement d'une climatologie étendue. Son utilisation opérationnelle couvre aujourd'hui les domaines du suivi de la ressource en eau, de la prévision des crues et de l'identification des sécheresses. Il se révèle également être un outil particulièrement adapté pour les études d'impact du changement climatique sur le cycle de l'eau.

\section{Abstract}

A hydrometeorological tool

for operational monitoring and research:

the Safran-Isba-Modcou

application (SIM)

The Safran-Isba-Modcou model combines an analysis system of the atmospheric forcing, a land surface scheme, and a hydrogeological model. Advanced research has been done around this suite during the last fifteen years. Since 2003, it has been transferred to Météo-France real-time operational environment and has been progressively supplied with an extended climatology. Its operational use now covers water resources monitoring, flood forecasting, and drought assessment. It is also particularly useful for studying the impact of climate change on the water cycle.
$\mathrm{U}$ ne bonne estimation des bilans d'eau et d'énergie à la surface (ainsi que les flux associés) est nécessaire pour la prévision numérique du temps et du climat. Les travaux de recherche de Météo-France dans ce domaine, menés depuis une vingtaine d'années, se sont principalement concrétisés par le développement et l'enrichissement progressif du modèle de surface Isba (Interactions solbiosphère-atmosphère).
Un des moyens de validation des simulations de bilans d'eau et d'énergie de surface est le couplage de modèles de surface et de modèles hydrologiques permettant de vérifier que les débits simulés des rivières sont cohérents avec les débits observés. C'est entre autres dans cet objectif que la chaîne SafranIsba-Modcou (SIM) a été initialement construite, en complément de l'intérêt de suivre les flux de surface et les réserves en eau du sol sur la France et d'en

Figure 1 - Schéma de fonctionnement de la chaîne Safran-Isba-Modcou (SIM).

\begin{tabular}{|c|c|}
\hline & Le système Safran-Isba-Modcou \\
Analyse & Analyses Arpège ou ECMWF \\
Safran & observations de surface \\
Forçage atmosphérique : pluie, neige, humidité, \\
rayonnements incidents, température, venties \\
Schémo de surfoce \\
Isba \\
$\begin{array}{c}\text { Photosynthèse, } \\
\text { végétation } \\
\text { interactive } \\
\text { pour le sol et la végétation }\end{array}$
\end{tabular}


connaître la climatologie ainsi que les valeurs au jour le jour pour l'initialisation des modèles atmosphérique et hydrologiques. Cette chaîne (figure 1) est composée d'un système d'analyse météorologique (Safran), du modèle de surface Isba et du modèle hydrogéologique Modcou de l'École des mines de Paris (Ledoux et al., 1989).

Cette chaîne a fait l'objet de nombreux travaux de validation par bassin à partir de 1995 sur l'Adour-Garonne (VoirinMorel, 2003), sur le Rhône (Etchevers et al., 2001) et la Seine (RoussetRegimbeau, 2007), puis d'une approche globale sur la métropole (Habets et al., 2008 ; Quintana Seguí et al., 2008). Elle a été portée dans l'environnement opérationnel de Météo-France fin 2003 et alimente les différentes bases de données de production. En outre, des réanalyses du modèle depuis 1970 et bientôt 1958, ont été également menées afin de disposer d'une climatologie spatialisée des paramètres hydrométéorologiques nécessaires au suivi de la ressource en eau en métropole.

Cet article présente les différents éléments de la chaîne SIM et ses principales applications pour la recherche et dans le domaine de la gestion de la ressource en eau des sols superficiels et des risques hydrologiques.

\section{De l'analyse des paramètres météorologiques aux débits des rivières}

Le système d'analyse Safran a été développé à l'origine par le Centre d'études de la neige (Durand et al., 1993) pour la prévision du risque d'avalanches (Safran signifie d'ailleurs « système d'analyse fournissant des renseignements à la neige »). Il a été étendu à l'ensemble du bassin du Rhône (Etchevers et al., 2001), puis à la France entière (Le Moigne, 2002). Safran analyse huit paramètres météorologiques au pas de temps horaire : le vent à 10 mètres, la température de l'air et l'humidité relative à 2 mètres, la nébulosité, les rayonnements solaires visible et infrarouge, ainsi que les précipitations liquides et solides.

Il s'appuie sur une méthode robuste d'interpolation optimale pour les principaux paramètres météorologiques. Son originalité provient du découpage en zones climatiques homogènes (les observations de zones voisines ont moins de poids que celles de la zone analysée) qui tient compte explicitement de l'altitude : pour une zone donnée, des analyses à différentes altitudes sont effectuées, avec une résolution verticale de 300 mètres. Dans l'application sur la France entière, c'est le zonage Symposium de Météo-France, découpant la France en environ six cents zones, qui a été utilisé. Le modèle Safran s'appuie à la fois sur l'ensemble des observations disponibles du réseau d'observations (précipitation, température...) ainsi que sur les analyses des modèles atmosphériques (Arpège ou CEPMMT) qui servent d'ébauche pour l'analyse. En outre, il estime les termes de rayonnement (visible et infrarouge) à partir d'un modèle radiatif utilisant en entrée les paramètres analysés (nébulosité et champs d'altitude).

Une validation de Safran a été effectuée récemment (Quintana Seguí et al., 2008). Cette étude a permis de confirmer la pertinence de ce module en matière d'analyse météorologique et notamment des champs de précipitation et de température de l'air, sans biais climatologiques (figure 2). Elle a aussi permis de mettre en évidence certains défauts qui pourront être corrigés dans les années à venir, en particulier sur la répartition horaire des précipitations (les observations horaires de précipitation ne sont pas utilisées par le système), ou le rayonnement. L'utilisation de données de rayonnement solaire observées au sol ou par satellite devrait permettre une meilleure estimation spatialisée du rayonnement pour les années les plus récentes. Dans le cadre de la chaîne SIM, les paramètres météorologiques analysés sont réinterpolés sur une grille régulière de $8 \mathrm{~km}$ x $8 \mathrm{~km}$ couvrant la France.

Le schéma de surface Isba (Noilhan et Planton, 1989 ; Noilhan et Mahfouf, 1996) a été conçu pour être utilisé dans les modèles de prévision numérique du temps et du climat et utilisait à sa conception un nombre très limité de variables pronostiques. Il a été par la suite progressivement enrichi. Dans l'application SIM, le modèle Isba est activé dans sa version hydrologique à trois couches (Boone et al., 1999) : couche superficielle, couche racinaire et couche profonde. Il intègre aussi un modèle d'évolution du manteau neigeux à trois couches (Boone et Etchevers, 2001) et des schémas de ruissellement et de drainage incluant une représentation sous maille de ces processus (Habets et al., 1999). Les paramètres décrivant la végétation et la texture du sol sont issus de la base Ecoclimap (Masson et al., 2003) à la résolution de $1 \mathrm{~km}$ sur la France. Isba étant un modèle physique, le seul paramètre de calage, le drainage sous maille, a été optimisé pour soutenir les débits d'étiage des cours d'eau. Dans le cadre de la chaîne SIM, Isba simule les bilans de surface à la résolution de $8 \mathrm{~km}$, et en particulier le ruissellement de surface et le drainage profond, nécessaires pour l'alimentation du modèle hydrologique.

Les transferts hydrologiques sont simulés par le modèle hydrogéologique Modcou, développé par l'École des mines de Paris (Ledoux et al., 1989) qui reçoit en entrée les données de ruissellement et d'infiltration issues d'Isba. Le ruissellement de surface est transféré

Figure 2 - Comparaison du cumul annuel de précipitation sur la France en 1974-1975 : a) établi à partir des postes de la BdClim, méthode Aurhely ; et b) analysé par Safran.

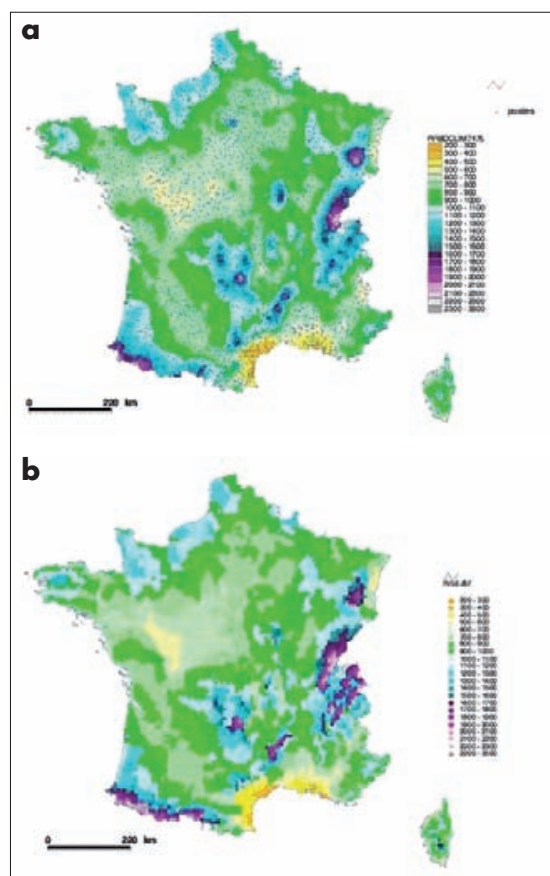

par le réseau hydrographique de surface. En présence d'une nappe souterraine, les flux de drainage permettent de calculer l'évolution spatiale et temporelle des niveaux des nappes. Modcou évalue ensuite les échanges entre les nappes et les rivières, puis calcule le routage en rivière suivant un algorithme simple reposant sur la détermination de zones isochrones. Dans la chaîne SIM, les débits sont calculés à un pas de temps de trois heures et l'évolution des hauteurs des nappes au pas de temps quotidien. Au niveau national, 
$42000 \mathrm{~km}$ de cours d'eau sont pris en compte et les calculs de débit sont disponibles sur plus de neuf cents points du réseau hydrographique (bassins versants entre $240 \mathrm{~km}^{2}$ et $112000 \mathrm{~km}^{2}$ ). Modcou représente actuellement de manière explicite les principales nappes du bassin versant de la Seine (trois nappes) et la nappe alluviale de la Saône et du Rhône.

Un exemple de validation de la chaîne SIM sur dix ans est décrit par Habets et al. (2008) par comparaison avec les débits observés, les hauteurs piézométriques des nappes et les observations du manteau neigeux. Cette étude a montré la bonne capacité du système à représenter la dynamique du bilan hydrique et des débits (débits moyens, mais aussi étiages et crues). En moyenne, sur six cents stations utilisées pour la comparaison, les débits simulés ne sont pas biaisés, et un tiers de ces stations simule de manière bonne ou très bonne l'évolution des écoulements (figure 3). Les simulations présentent des performances inférieures dans certains configurations identifiées : les bassins de taille inférieure à $1000 \mathrm{~km}^{2}$, les bassins fortement anthropisés (retenues hydroélectriques), les cours d'eau dont le régime est fortement influencé par les nappes lorsqu'elles ne sont pas explicitement prises en compte dans le modèle.

La chaîne SIM s'est révélée être un outil adapté à la simulation des épisodes de crue lente avec de bons scores statistiques pour la reproduction des débits de crue sur la Seine, tant au niveau du débit maximal observé que du phasage temporel du pic de crue (Rousset et al., 2004). Enfin, une étude récente du Centre national de recherche météorologique [CNRM] (Quintana Seguí et al., 2008) a montré qu'il était possible d'augmenter de manière significative

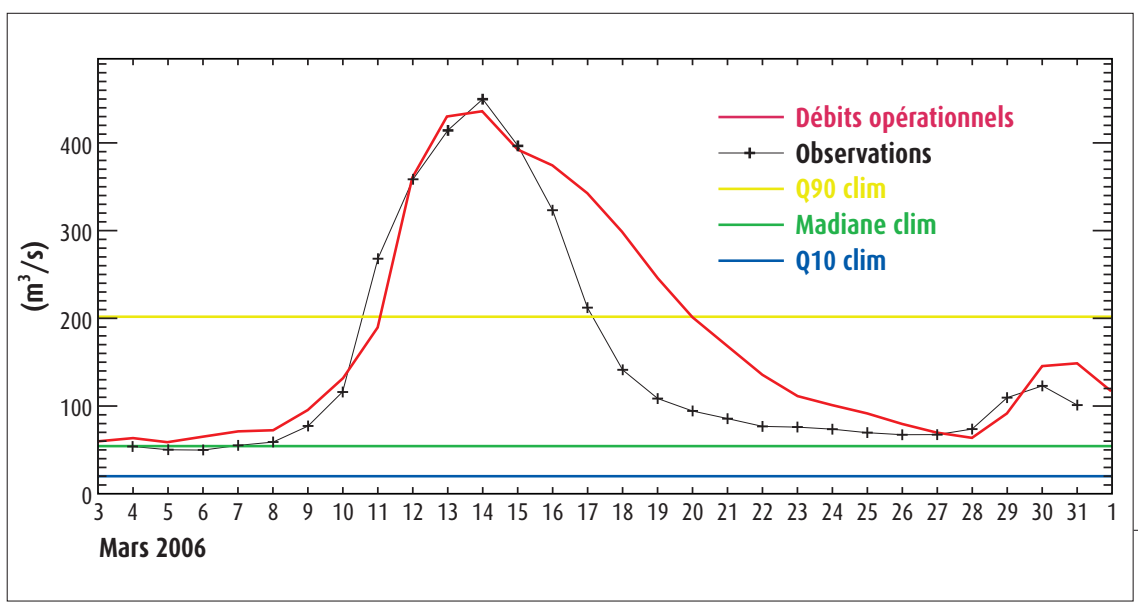

les performances de la chaîne SIM, en décrivant de manière plus détaillée les transferts hydriques et en tenant compte de la compaction du sol avec la profondeur (variation de la conductivité hydraulique à saturation en fonction de la profondeur du sol).

\section{Une combinaison opérationnelle des approches climatologiques en mode temps réel}

En mode opérationnel, la chaîne SIM fonctionne sur le supercalculateur NEC de Météo-France selon un fonctionnement temps réel (réseau automatique) et un mode réanalyse (la prise en compte du réseau climatique d'État permettant de tripler le nombre d'observations de précipitation) répondant aux besoins de recalage des systèmes hydrologiques et aux utilisations à visée climatologique.

En mode temps réel, la chaîne assure une analyse quotidienne sur le réseau de $06 \mathrm{~h}$ UTC (reconstitution de la période $\mathrm{J}-1,6 \mathrm{~h}$ à $\mathrm{J}, 6 \mathrm{~h}$ ) des variables météorologiques de surface en métropole à l'échelle de $8 \mathrm{~km}$ et utilise pour cela en particulier les données pluviométriques d'environ 1500 postes disponibles dans les bases opérationnelles. Il est envisagé d'utiliser à terme la lame d'eau Antilope (fusion lame d'eau radar et pluviomètres à résolution de $1 \mathrm{~km}$ ) pour améliorer la qualité de l'analyse des précipitations. Les sorties de Safran et Isba alimentent les bases de production, avec notamment des analyses horaires des différentes variables météorologiques de surface, mais aussi, par exemple, de l'humidité des sols, de l'épaisseur et de l'équivalent en eau du manteau neigeux. Le module Modcou estime pour sa part les débits moyens quotidiens sur les neuf cents points de jaugeage, en mode préopérationnel.

En mode réanalyse, un nouveau run de la chaîne est réalisé à une fréquence mensuelle pour bénéficier de la meilleure information disponible en matière d'observation pluviométrique : près de 4000 postes du réseau climatologique d'État sont alors pris en compte. Cette nouvelle analyse permet de recaler mensuellement la chaîne hydrologique opérationnelle et d'assurer un archivage de qualité optimale des analyses hydrométéorologiques de surface pour les études climatologiques.

Une reconstitution des données passées depuis 1970 (et bientôt 1958) a été réalisée en utilisant les champs météorologiques des archives opérationnelles et de la réanalyse ERA40 du Centre européen pour les prévisions météorologiques à moyen terme [CEPMMT] et des différentes données climatologiques archivées dans les bases de données de Météo-France. Ainsi, la chaîne SIM bénéficie aujourd'hui d'une climatologie étendue à $8 \mathrm{~km}$ de résolution spatiale sur la France, permettant de comparer en temps réel chaque situation quotidienne aux valeurs normales de saison ou à une année particulière. Pour faciliter l'accès à cette réanalyse aux différents utilisateurs hydrologiques, des produits quotidiens sont en cours de constitution et d'archivage dans la base climatologique de Météo-France et un accès standard via le service Climathèque du site Internet de Météo-France pourra être bientôt proposé.

En mode expérimental depuis le début de l'année 2008, une chaîne SIM couplée à la prévision d'ensemble météorologique du CEPMMT (RoussetRegimbeau et al., 2007) permet de calculer des prévisions ensemblistes de débit à échéance de dix jours sur neuf cents points de mesures. Sur chaque point, cinquante et une prévisions de débit traduisent les évolutions possibles des débits en fonction des incertitudes des scénarios de précipitation. Cette application est surtout destinée à être utilisée sur des bassins de grande taille (supérieure à $1000 \mathrm{~km}^{2}$ ). Un développement est en cours pour inclure dans cette chaîne probabiliste les prévisions d'ensemble Arpège. 


\section{Des applications hydrométéorologiques essentielles pour la gestion de la ressource en eau et des risques hydrologiques}

La chaîne SIM est un outil très utile pour les applications hydrométéorologiques opérationnelles car elle permet de disposer en temps réel et différé d'une information spatialisée continue et uniforme sur le territoire, à une résolution fine de $8 \mathrm{~km}$ et spécialement riche tant en variables météorologiques qu'hydrologiques.

En 2004, une expérimentation des produits au Service central d'hydrométéorologie et d'appui aux inondations [Schapi] a été mise en place, tant pour l'analyse « temps réel » des situations propices aux crues que pour la constitution d'abaques visant à la qualification du risque de crues et la fourniture d'informations permettant l'initialisation d'autres modèles hydrométéorologiques.

Dans ce cadre, le Schapi a été également associé aux tests de la chaîne SIM expérimentale de prévision d'ensemble de débits, dont les données sont visualisables sur un site extranet dédié. (figure 4).

Des sorties graphiques, inspirées des travaux similaires au sein de la communauté hydrologique (initiative internationale Hepex, projet européen Efas mené au Joint Research Center), visent à synthétiser l'information des différents scénarios et à la traduire en matière de risque de dépassement de seuils. Des travaux de recherche en cours au CNRM ont montré que l'utilisation des prévisions d'ensemble à courte échéance du modèle Arpège (Thirel et al., 2008) améliorent les performances de la chaîne à courte échéance (celles correspondant aux préoccupations opérationnelles majeures de sécurité publique). Une assimilation des débits passés dans la chaîne est en cours de mise au point, dans le but de fournir un meilleur état initial au modèle hydrologique.

Les produits issus de la chaîne SIM présentent également un grand intérêt pour le suivi de la ressource en eau. Le ministère en charge de l'Environnement a d'abord souhaité, dès 2005, disposer

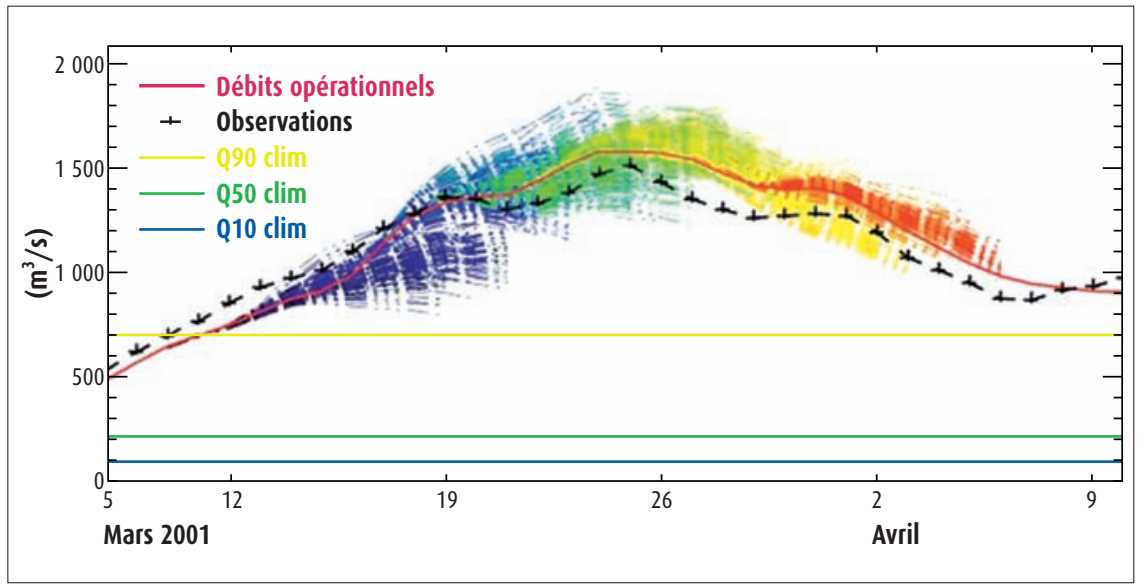

Figure 4 - Prévision d'ensemble des débits à dix jours sur la Seine à Paris pour la crue de mars 2001.

du produit « neige » (fraction de maille couverte de neige et équivalent en eau du manteau neigeux) de la chaîne SIM, dans le cadre de l'élaboration du Bulletin de suivi hydrologique national, afin de caractériser le potentiel de fonte nivale printanière sur les massifs montagneux des Alpes et des Pyrénées. Cette utilisation de la chaîne SIM s'est enrichie rapidement de la fourniture de l'humidité des sols (estimation de la ressource en eau des sols superficiels), puis d'une cartographie des pluies efficaces (différences entre précipitation et évapotranspiration réelle). Cette information issue de la modélisation SIM (figure 5) a aussi trouvé tout naturellement sa place dans le cadre du bilan régulier réalisé par le Comité national des effets de la sécheresse, présidé par le ministère de l'Écologie, de l'Énergie, du Développement durable et de l'Aménagement du territoire [Meeddat].

Le lien est étroit entre les situations d'étiage et les situations de sécheresse sévère des sols. Ces sécheresses sont à l'origine de dégâts importants aux bâtis du fait des phénomènes de retraitgonflement des argiles. Elles représentent un des chapitres les plus lourds de l'actuel dispositif national d'indemnisation des catastrophes naturelles. La chaîne SIM dispose d'atouts importants pour diagnostiquer ces phénomènes de sécheresse (Blanchard et al., 2007) et fait actuellement l'objet d'une évaluation interdisciplinaire avec les experts en géotechnique des sols dans le cadre du projet $\operatorname{Argic}^{(1)}$, visant à mieux comprendre les processus de subsidence touchant les argiles. À moyen terme, elle pourra être adaptée pour mieux décrire les transferts d'humidité dans les différentes couches de sol et apporter un diagnostic sur le risque de déformation, moyennant la poursuite des efforts de recherche.
Au-delà des applications hydrologiques mentionnées ici, les produits opérationnels issus de SIM servent quotidiennement de paramètres d'entrée à d'autres applications opérationnelles météorologiques : indice de risque de feux de forêt, modèle de prévision de température de surface de chaussée ou outil d'aide à l'alerte sur dépassement de seuils hydrométéorologiques. Des recherches ont montré son intérêt pour initialiser les réservoirs du sol des modèles atmosphériques de mésoéchelle Méso-NH et Arome. SIM reste aussi un cadre idéal pour évaluer le potentiel scientifique associé aux modèles de surface Isba et Teb (modèle de ville) et développer l'assimilation de données satellitaires pour les surfaces continentales.

\section{Un outil adapté aux études d'impact du changement climatique}

La question des impacts du changement climatique sur la disponibilité de la ressource en eau et des risques hydrologiques (sécheresse ou inondation) est un sujet de préoccupation majeur, confirmé en 2007 par le dernier rapport d'évaluation du Giec et la Loi sur l'eau et les milieux aquatiques (Lema).

La chaîne SIM, du fait de sa capacité à représenter l'intégralité du cycle de l'eau à l'échelle des bassins versants de taille moyenne à grande taille et son couplage avec les modèles météorologiques, est particulièrement pertinente

(1) Argic: Analyse du retrait-gonflement et de ses incidences sur les constructions. 
pour une application dans le cadre des études régionales d'impact dans le domaine de l'hydrologie.

Plusieurs études ont été menées ces dernières années sur différents bassins, en particulier sur le Rhône (Etchevers et al., 2002), la Garonne (Caballero et al., 2007) et la France (Boé et al., 2008). Une étude sur la Seine est menée actuellement avec SIM dans le cadre d'un projet du programme Gestion et impact du changement climatique (GICC) du Meeddat. Malgré les incertitudes des scénarios climatiques portant sur l'évolution du régime pluviométrique annuel, ces études montrent tout d'abord la forte sensibilité du manteau neigeux à toute augmentation de température. Cela se traduit par une modification du régime des rivières influencées par la neige : avancée du maximum de débit due à la fonte de la neige au printemps, augmentation de la durée de l'étiage estival (et baisse associée des débits), augmentation des débits hivernaux dus à l'augmentation de la part des précipitations pluvieuses. En dehors des phénomènes liés au manteau neigeux, l'augmentation de l'évapotranspiration dans un climat plus chaud se traduit par une baisse des débits, marquée en particulier l'été, et identifiant des problèmes potentiellement importants de gestion des ressources en eau.
Deux points méritent d'être soulignés. D'une part, il est nécessaire d'améliorer les techniques de descente d'échelle, opération nécessaire pour passer de l'échelle des modèles de climat à celle de la chaîne SIM. Les travaux récents menés au Centre européen de recherche et de formation avancée en calcul scientifique [Cerfacs] (Boé, 2007) ou dans le cadre de projets tels que Cyprim (Martin et al., 2007) sont très encourageants et ouvrent de nouvelles perspectives, en particulier pour l'étude des phénomènes extrêmes. D'autre part, il est nécessaire en parallèle de poursuivre l'amélioration du modèle par l'intégration de nouvelles nappes souterraines, l'adoption d'un nouveau schéma de routage en rivière ou encore une meilleure description de la zone non saturée. En outre, la prise en compte d'une paramétrisation de la photosynthèse tenant explicitement compte des variations de teneur en $\mathrm{CO}_{2}$ de l'atmosphère ainsi qu'une description multicouche du sol plus détaillée qu'aujourd'hui feront partie des futures évolutions du modèle.

La qualité de SIM pour la représentation à résolution fine du climat actuel représente un atout majeur pour les études d'impact du changement climatique.
Une étude financée par la fondation MAIF (projet ClimSec) est notamment en cours à Météo-France sur les impacts du changement climatique sur la sécheresse et l'eau du sol (Vidal et Soubeyroux, 2008). Elle pourra permettre de caractériser, à l'échelle régionale, l'évolution des caractéristiques des sécheresses au cours du XXI ${ }^{e}$ siècle, en fonction des divers scénarios du Giec sur les émissions de gaz à effet de serre.

\section{Conclusion}

L'application Safran-Isba-Modcou est un outil faisant l'objet de recherches et d'améliorations constantes depuis une quinzaine d'années et bénéficie d'une large reconnaissance dans la communauté hydrométéorologique. Son utilisation récente pour les applications opérationnelles présente un grand potentiel dans des domaines stratégiques de l'action des services de l'État (gestion de la ressource en eau et des risques).

Les développements en cours en matière de production et d'accès aux données permettront prochainement d'accroître l'utilisation et la valorisation de ces données pour les bureaux d'étude et les laboratoires scientifiques.

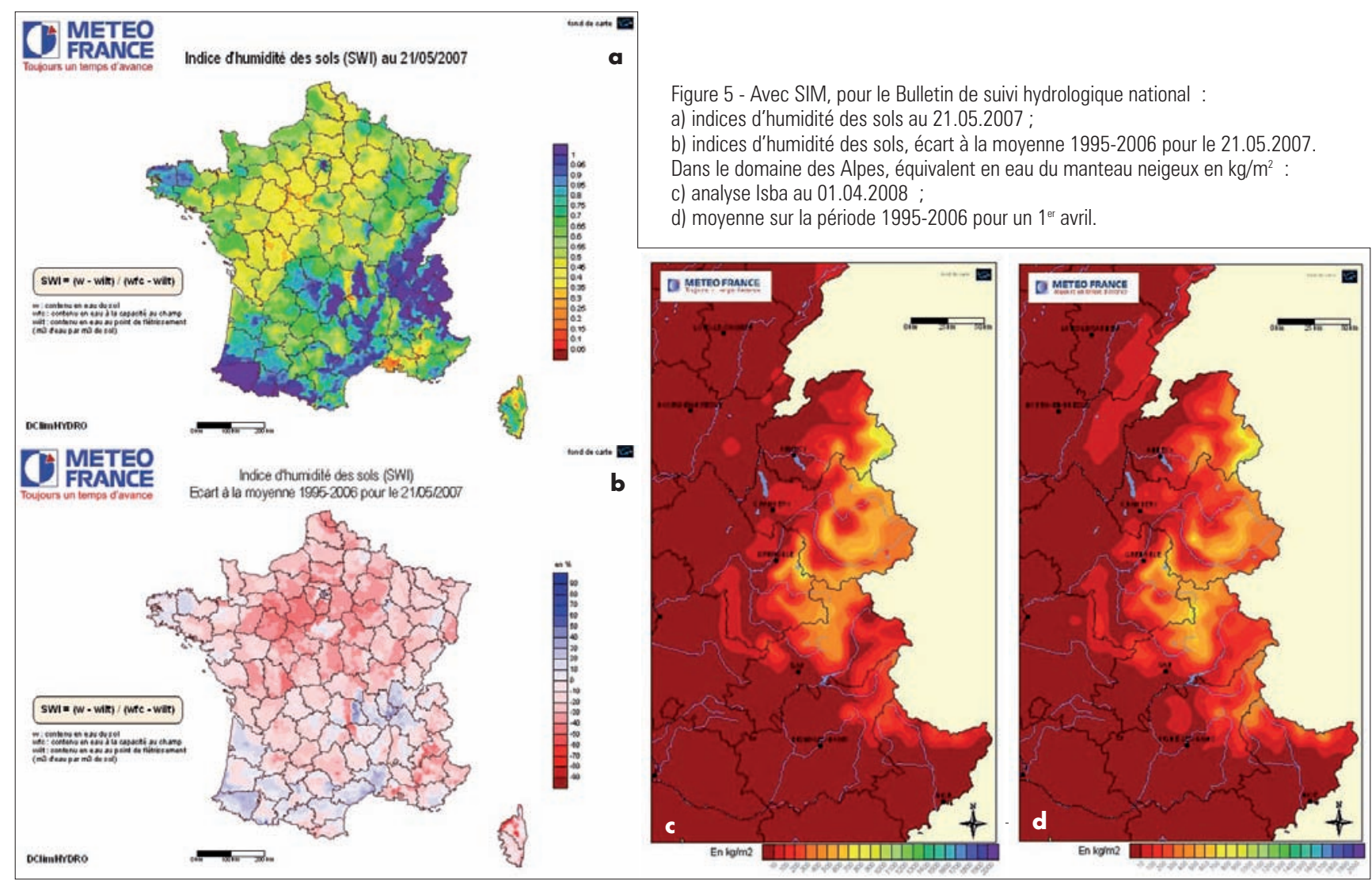


Ce transfert réussi entre le monde de la recherche et les services de production sera poursuivi au cours des prochaines années grâce à un programme important d'études autour de l'amélioration de l'analyse Safran, de la description des processus d'infiltration de l'eau dans le sol dans Isba, de l'amélioration des nappes dans Modcou et du routage de l'eau dans les rivières, en collaboration avec l'École nationale supérieure des mines de Paris [ENSMP].

Une utilisation temps réel de la chaîne SIM pour la prévision des crues pourra aussi être envisagée à terme grâce à la mise en place d'une assimilation des débits en temps réel et un couplage opérationnel avec des prévisions d'ensemble météorologiques.

Tous ces chantiers, qui mobilisent les équipes de recherche comme les services opérationnels, placent aujourd'hui la chaîne SIM comme un produit de référence en matière de modélisation hydroclimatique à l'échelle française et même européenne avec de nombreux échanges dans le cadre de projets européens tels Showcase-Eurogrid de l'European Climate Support Network (ECSN) ou le programme SAF Hydro (Satellite Application Facilities) de l'organisation Eumetsat.

\section{Remerciements}

Ces travaux ont été réalisés dans un cadre partenarial de recherche avec de nombreux laboratoires. Les auteurs souhaitent remercier plus particulièrement le PNRH et le GICC pour leur soutien et les laboratoires du CNRS (LTHE, CETP), du BRGM et du Cemagref pour leur contribution ainsi que Pierre Bessemoulin et Jean-Paul Goutorbe de Météo-France, qui ont joué un rôle décisif au démarrage du projet.

\section{Bibliographie}

\footnotetext{
- Blanchard M., L. Franchistéguy, F. Habets, E. Martin et J. Noilhan, 2007 : Typologie des sécheresses sur la France et outils de suivi de la ressource en eau utilisés à - Météo-France. Revue française de géotechnique, 120-121, 11-21, [http://hal-meteofrance.archives-ouvertes.fr/meteo-00274453/fr/].

- Boé J., L. Terray, F. Habets et E. Martin, 2006 : A simple statistical-dynamical downscaling scheme based on weather types and conditions resampling. J. Geophys. Res., 111, D23106

- Boé J., L. Terray, E. Martin et F. Habets, 2008 : Impacts of climate change on the hydrological cycle methodological issues and application to France. Water Resour. Res. soumis.

Boone A., J.-C. Calvet et J. Noilhan, 1999 : Inclusion of a third soil layer in a land surface scheme using the force-restore method. J. Appl. Meteor., 38, 1611-1630.

- Boone A. et P. Etchevers, 2001 : An inter-comparison of three snow schemes of varying complexity coupled to the same land-surface model: Local scale evaluation at an Alpine site. J. Hydrometeorol., 2, 374-394.

- Caballero Y., S. Voirin-Morel, F. Habets, J. Noilhan, P. Le Moigne, A. Lehenaff et A. Boone, 2007 : Hydrological sensitivity of the Adour-Garonne river basin to - climate change. Water Resour. Res., 43, W07448.

- Durand Y., E. Brun, L. Mérindol, G. Guyomarc'h, B. Lesaffre et E. Martin, 1993 : A meteorological estimation of relevant parameters for snow models. Ann. Glaciol., 18, 65-71.

- Etchevers P., C. Golaz et F. Habets, 2001 : Simulation of the water budget and the river flows of the Rhone Basin from 1981 to 1994. J. Hydrol., $224,60-85$.

- Etchevers P., C. Golaz, F. Habets et J. Noilhan, 2002 : Impact of a climate change on the Rhone river catchment hydrology. J. Geophys Res., 107, D16, 4293.

- Habets F., P. Etchevers, C. Golaz, E. Leblois, E. Ledoux, E. Martin, J. Noilhan et C.Ottlé, 1999 : Simulation of the water budget and the river flows of the Rhône basin. J. Geophys. Res., 104, 31145-31172.

- Habets F., A. Boone, J.-L. Champeaux, P. Etchevers, L. Franchistéguy, E. Leblois, E. Ledoux, P. Le Moigne, E. Martin, S. Morel, J. Noilhan, P. Quintana - Segui, F. Rousset-Regimbeau et P. Viennot, 2008 : The Safran-Isba-Modcou hydrometeorological model applied over France. J. Geophys. Res., 113 , D06113.

- Ledoux E., G. Girard, G. de Marsily et J. Deschenes, 1989 : Spatially distributed modeling: Conceptual approach, coupling surface water and ground-water, in - Unsaturated Flow Hydrologic Modeling - Theory and Practice. NATO ASI Series C, vol. 275, édité par H. J. Morel-Seytoux, p. 435-454, Kluwer Acad., Norwell, Massachussetts, États-Unis.

- Le Moigne P., 2002 : Description de l'analyse des champs de surface sur la France par le système Safran. Note de centre GMME, Météo-France.

- Martin E., V. Ducrocq, A. Joly, B. Joly, B. Nuissier, P. Quintana Seguí, D. Ricard, F. Sevault, S. Somot et P. Drobinski, 2007 : La Méditerranée, région témoin - de Cyprim à Hymex. La Houille blanche, 6, 90-96.

- Masson V., J.-L. Champeaux, F. Chauvin, C.Meriguet et R.Lacaze, 2003 : A global database of land surface parameters at $1 \mathrm{~km}$ resolution in meteorological and - climate models. J. Clim., 16, 1261-1282.

- Noilhan J. et S. Planton, 1989 : A simple parameterization of land surface processes for meteorological models. Mon. Weather Rev., 117, 536-549.

- Noilhan J. et J.-F. Mahfouf, 1996 : The Isba land surface parameterization scheme. Global Planet. Change, 13, 145-159.

Quintana Seguí P., P. Le Moigne, Y. Durand, E. Martin, F. Habets, M. Baillon, L. Franchistéguy, S. Morel et J. Noilhan, 2008 : Analysis of near surface atmosphe- ric variables: validation of the Safran analysis over France. J. Appl. Meteor. Climatol., 47, 92-107.

Rousset F., F. Habets, E. Gomez, P. Le Moigne, S. Morel, J. Noilhan et E. Ledoux, 2004 : Hydrometeorological modeling of the Seine basin using the Safran-Isba- Modcou system. J. Geophys. Res., 109, D14105.

- Rousset-Regimbeau F., 2007 : Modélisation des bilans de surface et des débits sur la France, application à la prévision d'ensemble des débits. Thèse de doctorat, 215 p., - université Paul-Sabatier, Toulouse, France.

Rousset-Regimbeau F., F. Habets, E. Martin et J. Noilhan, 2007 : Ensemble Streamflow forecasts over France. ECMWF Newsletter, $111,21-27$.

- Thirel G., F. Rousset-Regimbeau, E. Martin et F. Habets, 2008 : On the differences between the short-range forecasts of two Ensemble Streamflow Prediction - Systems. J. Hydromet., accepté.

- Vidal J.-P. et J.-M. Soubeyroux, 2008 : Impact du changement climatique sur la sécheresse et l'eau du sol. SEC 2008 - International Symposium - Drought and - Constructions (J.-P. Magnan, R. Cojean, Y.-J. Cui et P. Mestat, eds.), vol. 1, 25-31.

- Voirin-Morel S., 2003 : Modélisation distribuée des flux d'eau et d'énergie et des débits à l'échelle régionale du bassin Adour-Garonne. Thèse de doctorat, 292 p. - université Paul-Sabatier, Toulouse, France.
} 